\title{
Reactive Oxygen Species within the Spinal Cord Impairs Arterial Baroreflex Control of Renal Sympathetic Nerve Activity
}

\author{
Maycon Igor de Oliveira Milanez, Cássia Toledo Bergamaschi, Ruy Ribeiro Campos and Erika Emy Nishi*
}

Department of Physiology, Cardiovascular Division, Universidade Federal de São Paulo, Brazil

Received: October 10, 2017; Accepted: November 03, 2017; Published: November 19, 2017

*Corresponding author: Erika E. Nishi, Department of Physiology, Cardiovascular Division, Universidade Federal de São Paulo, Brazil, Tel: +55 11 5576 4848; E-mail: enishi@unifesp.br

\begin{abstract}
Clinical and experimental studies have shown that sustained increase in renal sympathetic nerve activity (rSNA) contributes to hypertension. Previous studies by our group showed that antioxidant treatments improve arterial baroreceptor reflex, which is a powerful beat-to-beat negative feedback control of arterial blood pressure (BP). We hypothesized that reactive oxygen species (ROS) contribute to arterial baroreflex control of rSNA by acting on sympathetic preganglionic neurons in the spinal cord. We then performed the intrathecal (i.t.) administration of tempol (4-hydroxy-2, 2, 6, 6-tetramethylpiperidine- $\mathrm{N}$-oxyl) ( $5 \mathrm{nmol})$, a superoxide dismutase mimetic. We evaluated the cardiovascular effects, basal and reflex rSNA evoked by i.t. injection of tempol. No significant changes in basal levels of heart rate (HR), mean arterial pressure (MAP) and rSNA were found. However, tempol significantly increased renal reflex sympathoinhibitory responses (pre tempol: $-0.61 \pm 0.15$; post tempol: $-1.55 \pm 0.14^{*}$ spikes $/ \mathrm{s} / \mathrm{mmHg}$ ). Thus, the results suggest that ROS exert a tonic inhibitory influence on the activity of spinal neurons with barosensitive properties, and it seems to be a preferential influence on fibers involved in reflex sympathoinhibitory responses. Our study raises the possibility that ROS in the spinal cord mediate part of the baroreflex dysfunction in cardiovascular diseases, such as hypertension and heart failure, which is detrimental to patient outcome.
\end{abstract}

Keywords: spinal cord; renal sympathetic activity; arterial baroreflex; reactive oxygen species;

\section{Introduction}

\section{Spinal Reactive Oxygen Species Contributes to Renal Baroreflex Control}

The arterial baroreceptor reflex is a powerful beat-tobeat negative feedback control of arterial blood pressure. Baroreceptors are mechanoceptors located in the aortic arch and at the bifurcation of the carotid arteries, being sensitive to the stretching of such vessels when arterial pressure is changed. Signals from these structures are centrally integrated, evoking changes in the pattern of sympathetic vasomotor activity controlling BP (Vasquez et al., 1997; Campos et al., 2001). In order to clarify the physiological aspects of the respective reflex, the brain circuitry involved in the modulation of this mechanism has been extensively studied, as well as its main neurotransmitters (Gordon, 1987; Lawrence e Jarrott, 1994; Tan et al., 2007). Moreover, the existence of spinal neurons with barosensitive properties has been described, bringing to light the important role of the spinal cord in the reflex control of BP (Mccall et al., 1977; Campos et al., 2017). However, the spinal cord mechanisms involved in this transmission need to be clarified.

Previous studies point to the involvement of reactive oxygen species (ROS) in cardiovascular diseases, and it seems to be an important molecular target to attenuate the systemic effects evoked by such disorders (Han et al., 2007; Nishi et al., 2013). Furthermore, evidence indicates that ROS, when in excess on the system, have the ability to influence baroreflex by attenuating the mechanism sensitivity, suggesting that the existence of oxidative balance is crucial for normal reflex activity (Li et al., 1996; Campos et al., 2015). In fact, our group showed that treatment with Vitamin C for seven consecutive days significantly increased baroreceptor sensitivity not only in animals with renovascular hypertension, but also in normotensive animals (Nishi et al., 2010). Corroborating with these findings, another study showed that the reduction of ROS in the rostral ventrolateral medulla (RVLM) was able to increase the sensitivity of the baroreflex response in spontaneously hypertensive stroke prone rats (Ogawa et al., 2012). For more details about the neural control of BP we recommended the review wrote by Thomas (2011) (Thomas, 2011). Little is known about the physiological role played by spinal ROS on basal and reflex modulation of rSNA. Therefore, the present study aimed to evaluate the cardiovascular responses and the rSNA evoked by the i.t. administration of tempol, a superoxide dismutase (SOD) mimetic. In addition, we assessed the arterial baroreceptor reflex sensitivity to the renal territory after spinal administration of tempol, investigating a possible participation of the spinal ROS in the reflex control of the sympathetic vasomotor activity to the renal bed. 


\section{Methods}

\section{Animals}

The experimental protocols employed in this study were performed under guidelines recommended by the National Institutes of Health and approved by the Ethics in Research Committee of the Escola Paulista de Medicina - Universidade Federal de São Paulo (process No.8724270715/15). Male Wistar rats $(n=4)(300-350 \mathrm{~g})$ were housed in group cages, given access to rat chow and water ad libitum, and maintained in a temperaturecontrolled environment $\left(23^{\circ} \mathrm{C}\right)$ with a 12 -hour light/dark cycle.

\section{Recording of mean arterial pressure and heart rate}

Rats under ketamine (80-100 mg/kg, ip) and xylazine (10 $\mathrm{mg} / \mathrm{kg}$, ip) anesthesia had the femoral vein catheterized for intravenous injection of drugs and the femoral artery catheterized for direct recording of arterial pressure. After surgical recovery (approximately 24 hours), baseline pulsatile BP, MAP and HR were recorded in conscious rats (PowerLab -ADInstruments, Australia). Average values of basal MAP and HR were obtained by continuous recording for 10 minutes.

\section{Intrathecal Injection}

In urethane-anesthetized rats ( 1.2 to $1.4 \mathrm{~g} / \mathrm{kg}$ ) (SigmaAldrich, St. Louis, MO), the spinal subarachnoid space was cannulated for the i.t administration of drugs. The atlanto-occipital membrane was exposed by removing the overlying neck musculature through a midline dorsal incision. The head of the rat was mildly depressed and the membrane was carefully slit. A polyethylene (PE-10) catheter filled with tempol ( $5 \mathrm{nmol}$ in $2 \mu \mathrm{L}$ - SigmaAldrich, St. Louis, MO) dissolved in saline was advanced caudally in the spinal sub-arachnoid space until T11-12 segment of spinal cord, where the drug was injected. The drug effects were analyzed over 10 minutes; the validity of this method for correct positioning of the tip of the catheter was confirmed in post-mortem analysis.

\section{Analysis of renal sympathetic nerve activity (rSNA) and the arterial baroreflex control of rSNA in urethane anesthetized rats}

The left renal nerve was retroperitoneally exposed and placed on bipolar silver electrodes. The signal from the renal nerve was displayed on an oscilloscope TDS 220 (Tektronix, Beaverton, OR). The nerve activity was amplified (gain $20 \mathrm{~K}$ Neurolog; Digitimer, Welwyn Garden City, Herts, UK), filtered by a band-pass filter (100$1000 \mathrm{~Hz}$ ), and collected for display and subsequent analysis using a PowerLab data acquisition system (ADInstruments, Sydney, Australia). At the end of the experiments, the background noise level was determined by hexamethonium bromide administration (30 mg/kg, intravenously) (SigmaAldrich, St. Louis, MO). The neural activity was analyzed using the appropriate software spike histogram and was expressed as spikes/second (ADInstruments).

For analysis of the arterial baroreflex control of rSNA, bolus injections $(0.1 \mathrm{ml})$ of phenylephrine $(10 \mu \mathrm{g}$, iv) (SigmaAldrich, St.
Louis, MO) and sodium nitroprusside (20 $\mu \mathrm{g}$, iv) (SigmaAldrich, St. Louis, MO) were administered pre and post i.t. injection of tempol. Values of matching MAP variations ( $\triangle$ MAP from 5 to $40 \mathrm{~mm} \mathrm{Hg})$ with reflex rSNA $(\triangle \mathrm{rSNA})$ responses were plotted separately for each vasoactive drug to measure baroreceptor function, and their gain (spikes/s/mmHg) were compared to evaluate changes in arterial baroreflex sensitivity.

\section{Data Analysis}

The results are presented as the mean \pm SD. The data were evaluated by paired Student's t-test. The level of statistical significance was defined as $\mathrm{P}<0.05$.

\section{Results and Discussion}

The respective baseline values (HR, MAP and rSNA) of the animals are presented in the figure legends.

\section{Effects of intrathecal administration of tempol}

I.t. administration of tempol unchanged basal levels of HR, MAP and rSNA (Figure 1). However, tempol significantly increased renal reflex sympathoinhibitory responses evoked by intravenous injection of phenylephrine (pre tempol: $-0.61 \pm 0.15$; post tempol: $-1.55 \pm 0.14^{*}$ spikes/s/mmHg) (Figure 2), whereas no change occurred in sympathoexcitatory responses elicited by intravenous injection of sodium nitroprusside (pre tempol: 0.47 \pm 0.07 ; post tempol: $0.37 \pm 0.05^{*}$ spikes $/ \mathrm{s} / \mathrm{mmHg}$ ).
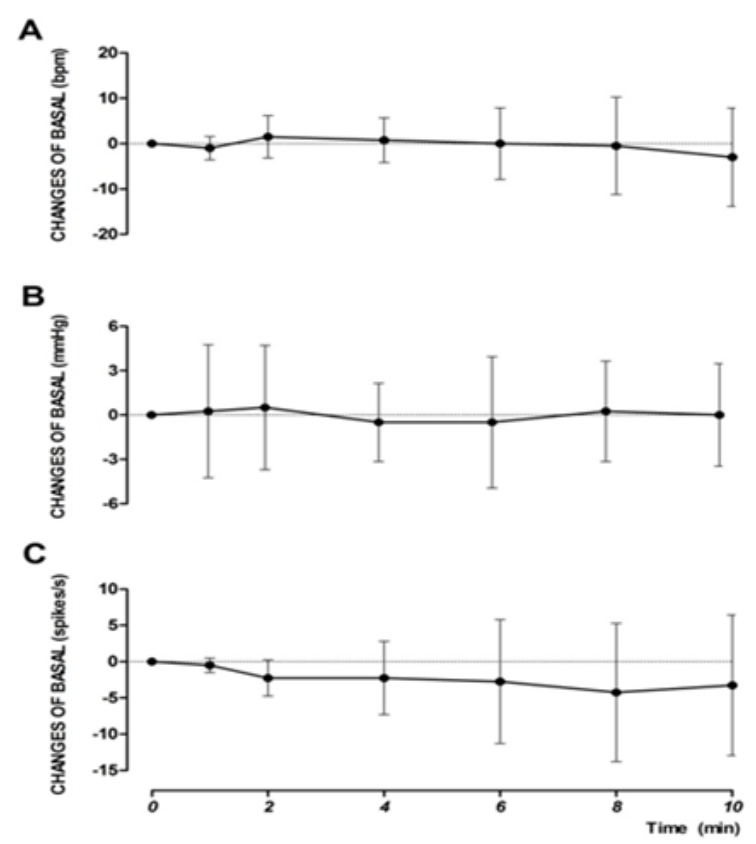

Figure 1: Responses evoked by intrathecal administration of tempol (5nmol) on heart rate ( $\mathrm{HR}, \mathrm{bpm})(\mathrm{A})$, mean arterial pressure (MAP, $\mathrm{mmHg}$ ) (B) and renal sympathetic nerve activity (rSNA, spikes/s) (C) presented as changes from baseline. Baseline values of HR, MAP and rSNA are: $474 \pm 21 \mathrm{bpm}, 100 \pm 6 \mathrm{mmHg}, 111 \pm 9$ spikes/s. Intrathecal administration of the antioxidant did not trigger any robust change in the HR, MAP and rSNA. 


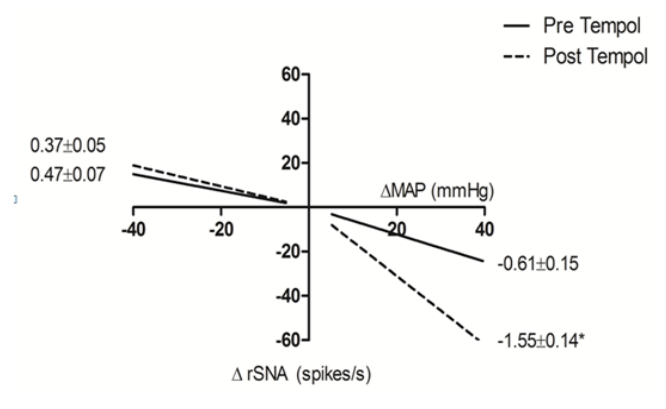

Figure 2: Analysis of the renal baroreceptor reflex. The figure shows that the intrathecal application of tempol triggers an increase in the gain of the sympathoinhibitory reflex response caused by phenylephrine. No significant change was observed regarding the sympathoexcitatory reflex responses. ${ }^{*} p<0.05$ (paired Student's t-test). Mean Arterial Pressure (MAP); Renal Sympathetic Nerve Activity (rSNA).
The results indicate a physiological role played by ROSs in the spinal cord regarding the control of rSNA. Although apparently not involved in the basal modulation of rSNA under physiological conditions, ROS appear to exert a tonic inhibitory influence on sympathetic preganglionic neurons with barosensitive properties in the spinal cord, apparently with a preferential influence on unmyelinated fibers involved in reflex sympathoinhibitory responses (Figure 3). Such findings suggest that, in addition to oxidative stress in specific brain areas involved in the control of sympathetic vasomotor tone (Ogawa et al., 2012; Campos et al., 2015), the spinal oxidative imbalance may also be contributing to the baroreceptor dysfunction seen in several cardiovascular diseases (Sawyer et al., 2002; Oliveira-Sales et al., 2008).

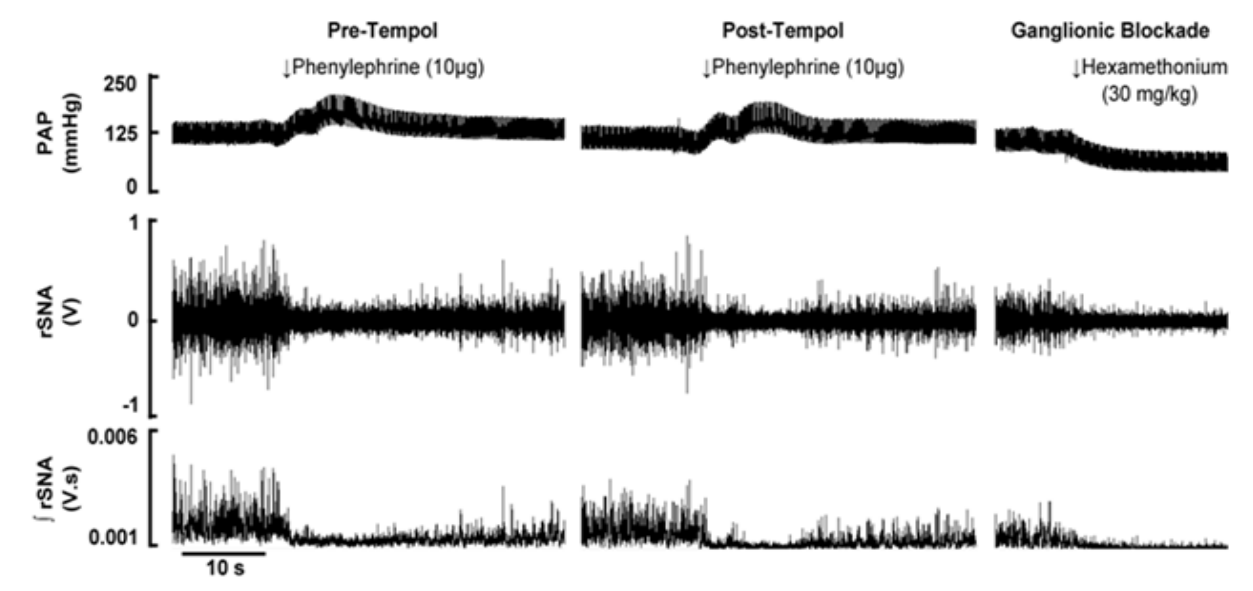

Figure 3: Representative image of the effect evoked by intrathecal administration of tempol on the reflex sympathoinhibition to the renal territory caused by the intravenous injection of phenylephrine. After the spinal application of the antioxidant, a more intense inhibitory effect is observed triggered by the pressor substance. These findings suggest that ROS exert an inhibitory effect on spinal neurons with barosensitive properties, especially those involved in reflex sympathoinhibition responses. Pulsatile Arterial Pressure (PAP); Renal Sympathetic Nerve Activity (rSNA).

Moreover, the results of the present study corroborate with recent findings obtained in our laboratory. We have shown that i.t. administration of losartan, an angiotensin II (Ang II) AT1 receptor antagonist, triggers a selective increase in sensitivity for renal sympathoinhibitory reflex responses in the renovascular hypertension model (Campos et al., 2017). The production of Ang II-mediated ROS is widely described in the literature (Seshiah et al., 2002; Han et al., 2007), therefore, it is reasonable to hypothesize that the baroreceptor dysfunction found in the respective hypertension model is evoked, in part, by a prooxidative spinal angiotensinergic action. However, further studies are needed to clarify the origin of spinal ROS, its topographic location, as well as the precursor agents involved in its synthesis.

Therefore, the fact that ROS contribute to arterial baroreflex control of rSNA by acting on sympathetic preganglionic neurons in the spinal cord under physiological condition raises the possibility that spinal ROS mediate part of the baroreflex dysfunction that is detrimental to the outcome of hypertensive and heart failure patients.

\section{Financial Support}

FAPESP (2013/13332-1); CAPES (0523/2014) and CNPq (472613/2013-8). C.T.B. and R.R.C. are recipients of CNPq fellowships. E.E.N. was the recipient of FAPESP (2013/23741-6) and CAPES-PNPD Postdoctoral Fellowships.

\section{References}

1. Campos Junior R, Colombari E, Cravo S, Lopes OU. Hipertensão arterial: o que tem a dizer o sistema nervoso. Rev Bras Hipertens. 2001;8(1):41-54.

2. Campos RR, Milanez MIO, Nishi EE, Bergamaschi CT. Influence of spi- 
nal neurotransmission on renal sympathetic nerve activity in renovascular hypertension. FASEB J. 2017;31(lb801).

3. Campos RR, Oliveira-Sales EB, Nishi EE, Paton JF, Bergamaschi CT Mechanisms of renal sympathetic activation in renovascular hypertension. Exp Physiol. 2015;100(5):496-501. doi: 10.1113/expphysiol.2014.079855

4. Gordon FJ. Aortic baroreceptor reflexes are mediated by NMDA receptors in caudal ventrolateral medulla. Am J Physiol 1987;252(3):628-633.

5. Han Y, Shi Z, Zhang F, Yu Y, Zhong MK, Gao XY, et al. Reactive oxygen species in the paraventricular nucleus mediate the cardiac sympathetic afferent reflex in chronic heart failure rats. Eur J Heart Fail. 2007;9(10):967-973. doi: 10.1016/j.ejheart.2007.07.004

6. Lawrence AJ, Jarrott B. L-glutamate as a neurotransmitter at baroreceptor afferents: evidence from in vivo microdialysis. Neuroscience. 1994;58(3):585-591.

7. Li Z, Mao HZ, Abboud FM, Chapleau MW. Oxygen-derived free radicals contribute to baroreceptor dysfunction in atherosclerotic rabbits. Circ Res. 1996;79(4):802-811.

8. McCall RB, Gebber GL, Barman SM. Spinal interneurons in the baroreceptor reflex arc. Am J Physiol. 1977;232(6):657-665.

9. Nishi EE, Bergamaschi CT, Oliveira-Sales EB, Simon KA, Campos RR. Losartan reduces oxidative stress within the rostral ventrolateral medulla of rats with renovascular hypertension. Am J Hypertens. 2013;26(7):858-865

10. Nishi EE, Oliveira-Sales EB, Bergamaschi CT, Oliveira TG, Boim MA, Campos RR. Chronic antioxidant treatment improves arterial renovascular hypertension and oxidative stress markers in the kidney in Wistar rats. Am J Hypertens. 2010;23(5):473-480. doi: 10.1038/ ajh.2010.11

11. Ogawa K, Hirooka Y, Shinohara K, Kishi T, Sunagawa K. Inhibition of oxidative stress in rostral ventrolateral medulla improves impaired baroreflex sensitivity in stroke-prone spontaneously hypertensive rats. International Heart Journal. 2012;53(3):193-198.

12. Oliveira-Sales EB, Dugaich AP, Carillo BA, Abreu NP, Boim MA, Martins PJ, et al. Oxidative stress contributes to renovascular hypertension. Am J Hypertens. 2008;21(1):98-104. doi: 10.1038/ajh.2007.12

13. Li Z, Mao HZ, Abboud FM, Chapleau MW. Oxygen-derived free radicals contribute to baroreceptor dysfunction in atherosclerotic rabbits. Circ Res. 1996;79(4):802-811.

14. Seshiah PN, Weber DS, Rocic P, Valppu L, Taniyama Y, Griendling KK. Angiotensin II stimulation of NAD(P)H oxidase activity: upstream mediators. Circ Res. 2002;91(5):406-413.

15. Tan PS, Killinger S, Horiuchi J, Dampney RA. Baroreceptor reflex modulation by circulating angiotensin II is mediated by AT1 receptors in the nucleus tractus solitarius. Am J Physiol Regul Integr Comp Physiol. 2007;293(6):2267-2278.

16. Thomas GD. Neural control of the circulation. Adv Physiol Educ. 2011;35(1):28-32. doi: 10.1152/advan.00114.2010

17. Vasquez EC, Meyrelles SS, Mauad H, Cabral AM. Neural reflex regulation of arterial pressure in pathophysiological conditions: interplay among the baroreflex, the cardiopulmonary reflexes and the chemoreflex. Braz J Med Biol Res. 1997;30(4):521-532. 